\title{
Back-Extraction Processes of C.C.Lipase with Mediated AOT Reverse Micellar System
}

\author{
Sung-Sik Lee, Bong-Gyu Kim, Nak-Chang Sung, and Jong-Pal Lee ${ }^{\ddagger}$ \\ Department of Chemical Engineering, Dong-4 Chiversitv Busan 604-714, Korea \\ ${ }^{\dagger}$ Deparment of Enirommental Engineering, Dong-A Chiversin. Busan 604-714, Korea \\ -Department of Chemistrv. Dong-A Universit, Busan 604-714. Korea \\ Received December 1, 2003
}

\begin{abstract}
The relationship between the behaviors of c.c.lipase back-extraction and their percolation phenomena by using AOT reverse micellar sy'stems (RVMS) has been studied by the addition of a small amount of additives to organic phase such as thiols and nonionic-surfactants focusing on micelle-micelle interactions. The values of $\beta$ defined by the variation of percolation processes and back-extraction behaviors of $c$ c.lipase have a good linear correlation. The hydrophobicity of additive molecules suppressing the cluster formation of reverse micelles (high values of $\beta$ ) improved the back-extraction behavior of c.c.lipase. The back-extraction fraction and its rate of c.c.clipase are increased with decreasing of the value of hydrophilic lipophilic balance (HLB) and increasing of the hydrophobicity per additive molecules added to reverse micellar șistems (RVSM) in the same additives concentration
\end{abstract}

Key Words : Back-extraction. Mediated AOT reverse miceller, C.c.lipase, Percolation

\section{Introduction}

The reverse micellar systems (RVMS) have a capability to solubilize a various biomolecules such as proteins and enzymes into the nanometer-size water pools surrounded by a monolayer of surfactant, which can be utilized for protein extraction and enzymatic reaction systems. Since it was discovered that proteins could be solubilized in reverse micelles. many researchers have studied on the protein extraction and enzymatic reactions by using RVMS ${ }^{1-12}$

Among various factors. $\mathrm{pH}$ and ionic strength are dominant factors for the reverse micellar extraction process. By controlling these parameters. the extracted fraction can be varied via variations of electrostatic. hydrophobic and steric interactions between proteins and micelles. Unfortunately. irreversible denaturation and inactivation of solubilized enzymes owing to the nonspecific interaction with micelles have been reported to be severe problems in the reverse micellar systems. One more severe problem caused by those is a notable decrease in recovered (backextracted) fraction and the back-extraction rate in reverse micellar extraction processes. This is also originated from the strong interactions between protein and micelle.

In our previous papers, ${ }^{13.14}$ we have shown that the alcohols suppressing the formation of micellar cluster. improved the back-extraction of BSA and cytochrome $\mathrm{c}$. Although the control of protem-micellar interactions is very difficult operation. these works have shown that it could be achieved by the addition of cosurfactant or other additives to modify the micelles if the additives were properly designed. For this reason. more simple and effective additives are

\footnotetext{
"To whom correspondence should be addressed. e-mail: ssleed daunet.donga.ac.kr
}

desired to be developed to modify the micelles. ${ }^{15}$

The percolation process reflects the formation of micellar cluster caused by the micellar-micellar interactions and it can be easily quantified by the measurement of electrical conductivity of the RVMS. Electrical conductivity measurements have been used to assess reverse micellar cluster formation and to probe the structural changes occurring in such systems. Individual droplets maintain a low conductivity. while a sharp increase in electrical conductivity is caused by the percolation phenomenon. well demonstrating the micellar-micellar interactions. It is generally accepted that the variation of the conductivity during percolation precesses in AOT-RVMS with a spherical droplet structure is a result of reverse micellar droplet clustering. The percolation threshold can be also varied by the addition of some molecules. but its effect has not been clearly understood yet.

In the present study, the behavior on back-extraction and their percolation phenomena of c.c.lipase by using AOTRVMS has been studied to focus on the formation of micellar cluster via protein-micelle interactions. And the relationship between the behaviors of c.c.lipase backextraction and their percolation phenomena has been studied by the addition of a small amount of co-surfactants such as thiols and nonionic surfactants. respectively.

\section{Experimental Section}

Materials. AOT(sodium di[2-ethylhexyl] sulfosuccinate) of purity $95 \%$ was purchased from Tokyo Kasei and used without further purification. C.c.lipase $(\mathrm{MW}=53 \mathrm{kDa}, \mathrm{pI}=$ 4.3) was purchased from Sigma. Ethanethiol (EtSH). 1propanethiol $(\mathrm{PrSH})$, 1-butanethiol $(\mathrm{BuSH})$, 1-pentanethiol (PenSH). 1-hexanethiol (HexSH), 1-heptanethiol (HepSH), 1.2-ethanedithiol $\left(\mathrm{Et}(\mathrm{SH})_{2}\right)$, 1.3-propanedithiol $\left(\mathrm{Pr}(\mathrm{SH})_{2}\right)$, 
1,4-butanedithiol $\left(\mathrm{Bu}(\mathrm{SH})_{2}\right), 1,5$-pentanedithiol $\left(\mathrm{Pen}(\mathrm{SH})_{2}\right)$ and 1.6-hexanedithiol $\left(\mathrm{Hex}(\mathrm{SH})_{2}\right)$ were purchased from Aldrich. Span85 was purchased from miyuchemical and PNIs ( $[: O=3,5,10,20 \mathrm{~mol}$ ) were purchased from Dongnam Chemical Industries ITD.

Methods. Percolation processes: The conductivity of RVMS was measured as a function of water content $\left(\phi_{\text {wit }}\right)$ with a conductivity meter CM-40V (TOA Flectronics L.td., Tokyo, Japan) and a platinum electrode. The electrode was inserted into the test tube containing the reverse micellar solution and the tube was placed in a thermostated water bath $\left(25 \pm 0.1^{\circ} \mathrm{C}\right)$. Flectrical conductivity measurements were performed with dropwise addition of an aqueous phase to AOT/isooctane or AOT-additive/isooctane solution until the percolation phenomenon was observed.

Back-extraction of c.lipase: The c.c.lipase was solubilized into AOT/isooctane solution by the injection method following the description in the previous paper. The buffer solution containing the c.c.lipase $(10 \mathrm{mM})$ was injected into AOT/isooctane solution ( $200 \mathrm{mM}$ AOT/isooctane, $\left.W_{0}=20\right)$ and the mixture as shaken vigorously until a clear solution was obtained. Back-extraction of the c.c.lipase from the reverse micelles was carried out by contacting the protein containing reverse micellar solution with buffer solution $\left(\mathrm{pH}_{\mathrm{aq}}\right)$ containing $0.1 \mathrm{M} \mathrm{KCl}$ for 3 hours reached an equilibrium state. Similar experiments were also carried out for AOT-additive mixed RVMS. The c.c.lipase backextraction behavior depends on the $\mathrm{pH}$ value in the feed solution injected into reverse micelles, $\mathrm{pH}_{\mathrm{inj}}$. The c.c.lipase concentrations were determined by spectrophotometer (UV$1600 \mathrm{~A}$, Shimadzu) at $280 \mathrm{~mm}$.

\section{Results and Discussion}

Effect of additive solubilization on the percolation processes of the AOT RVMSs. The percolation phenomenon of reverse micelles is changed by the solubilization of various materials (i.e. enzyme, protein and polymer). The percolation processes are effective for the evaluation of the micelle-Inicelle interactions. ${ }^{16-18} \mathrm{~A}$ sharp increase in electrical conductivity caused by the percolation process demonstrates well the existence of the strong micellar-micellar interaction inducing a micellar clustering. It is generally accepted that percolation in AOI-RVMS with a spherical droplet structure is a result of reverse micellar droplet clustering. ${ }^{18}$ The variation of the electrical conductivity of the $\mathrm{AO}$ ] reverse micellar solution is plotted in Figure 1 against the volume fraction of water in the organic phase, aq. In the case of thiols and spans solubilized into the RVMS, the electrical percolation threshold $\left(\phi_{1}\right)$ is increased with solubilizing thiols and spans into the reverse micelles. This result indicates an increase in the attractive interaction between micelles as thiols and spans solubilized into the RVMS. In contrast, for the RVMS solubilized PNEs, the percolation threshold ( $\phi_{1}$ ) was decreased in the lower value of $\phi_{\text {at }}$ than that of the additive-free system. 'These results suggest that the micelle-Inicelle interactions are notably influenced by

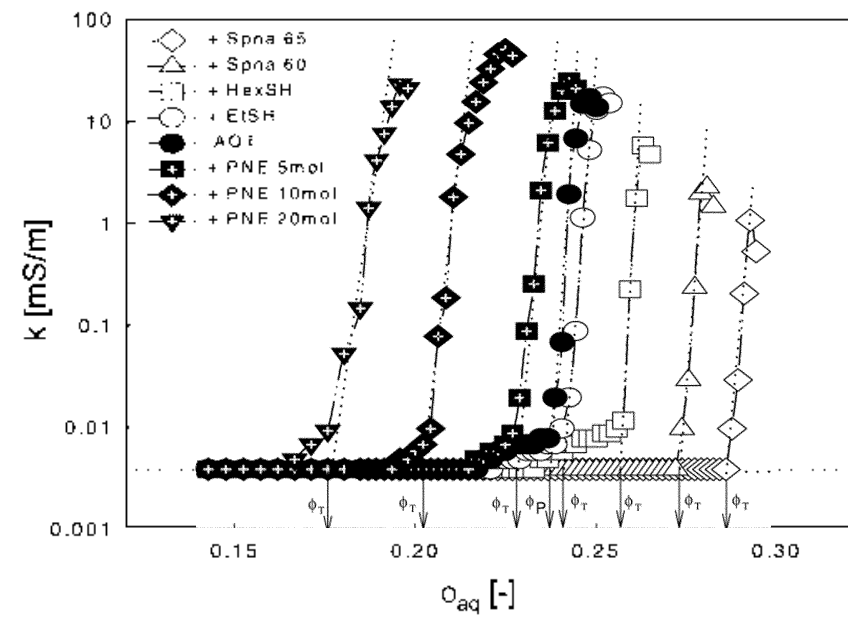

Figure 1. Variation of the percolation process of RVMS (AOT 200 m.M) with and without thiols. and nonionic surlactants.

the additive species and concentration solubilized into the reverse micelles. Proteins ${ }^{19.20}$ and additives ${ }^{17.21 .22}$ in reverse micelles has been studied by several authors. There are indications that cytochrome $\mathrm{c}$ interacts with the AOT surfactant layer. Therefore, the cytochrome c-micelle (electrostatic attractive) interactions seem to decrease the stability of RVMS by decreasing electrostatic repulsive interaction between micelles. ${ }^{19}$ The formation of micellar clusters shows a larger hydrophobic attraction than an electrostatic repulsive force between the micelles.

The difference, $\Delta \phi_{1}\left(=\phi_{1}-\phi_{1}\right)$, means the effect of the thiols, spans and PNTs concentration on the percolation process. Here, $\phi_{\mid}$and $\phi_{1}$ are the values of the percolation threshold with and without thiols, spans and PNEs, respectively. The relationship between relative percolation thresholds and added thiols concentrations is also examined. $\Delta \phi_{1}$ is plotted against the thiol concentration added in the reverse micellar solution in Figure 2. There is a linear correlation between $\beta$, and the concentration of each thiol. The slope, $\beta_{t}$, is a measure of the effect of thiol addition on the micellar-

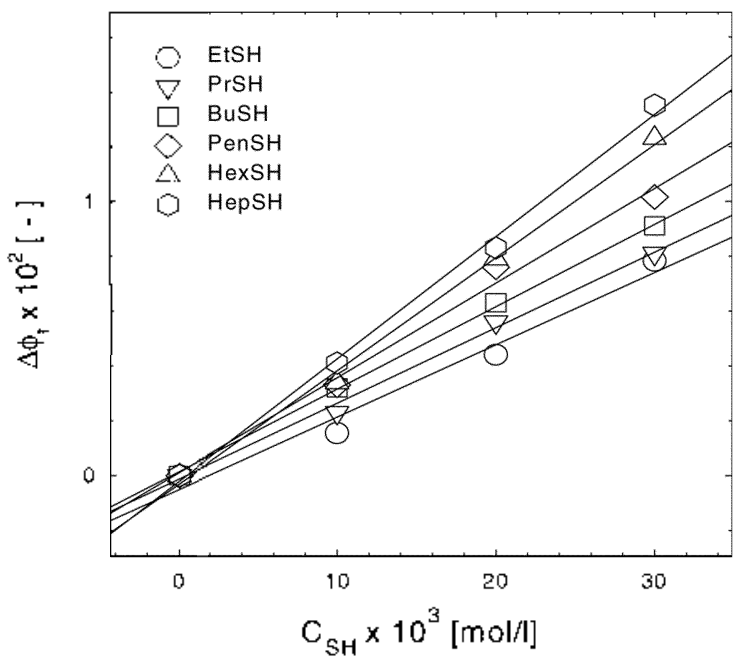

Figure 2. Liffect of additives. thiols on percolation process of RVMS. 


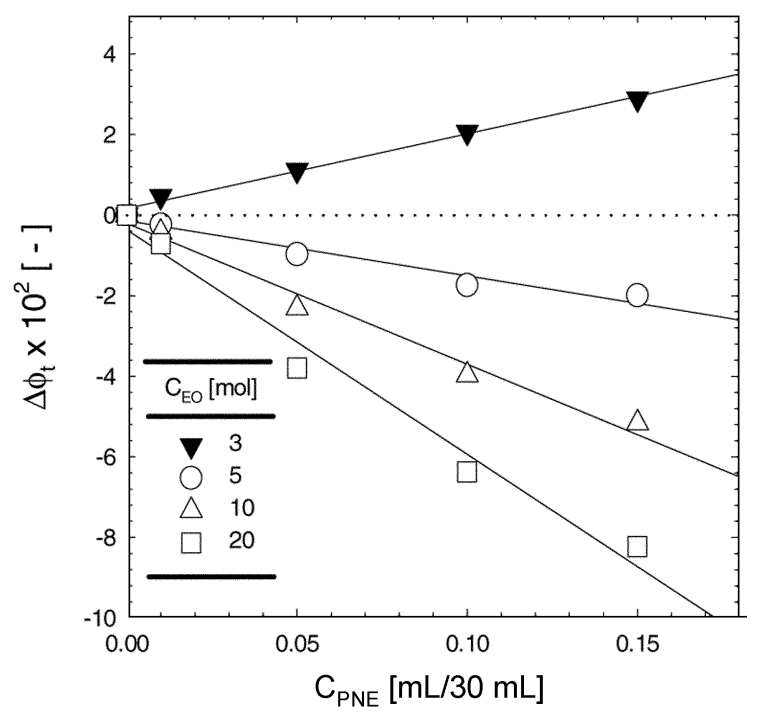

Figure 3. Effect of PVL concentration on the percolation behaviors of $\mathrm{CAB}$.

micellar interaction. The value of $\beta_{1}$ means the stability of RVMS or ability of water solubility with the addition of thiols. A positive value of $\beta_{1}$ means the stabilization of RVMS or the decrease of micellar-micellar interactions with the addition of thiols to RVMS. We have examined the effect of thiols on the micellar-micellar interaction using a percolation processes of RVMS and found that variation in these effects can be explained by the additive contribution of each constituent group of the thiol; the hydrocarbon $(\mathrm{CH})$ group makes a suppressive contribution and the hydrosulfon (SH) group contributes expeditiously to the effect. We have quantitatively evaluated these cosurfactant effects on the micellar-micellar interaction of RVMS (or micellar clustering) as values of $\beta_{1}$.

The relationship between the relative percolation thresholds and added nonionic-cosurfactant concentrations is also examined. $\Delta \Psi_{p}$ is plotted against the nonionic-cosurfactant

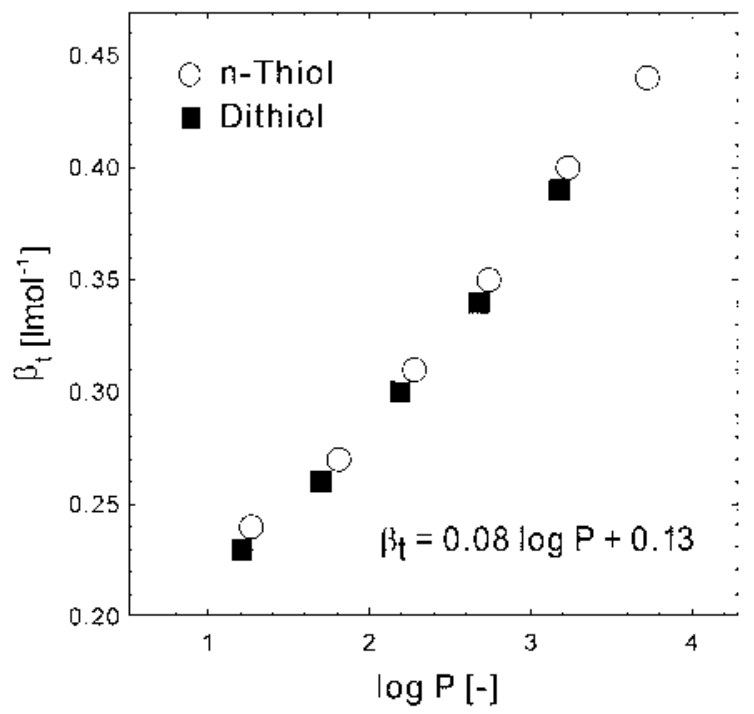

Figure 4. Correlation between the value of $\log P$ and $\beta$, of thiols.

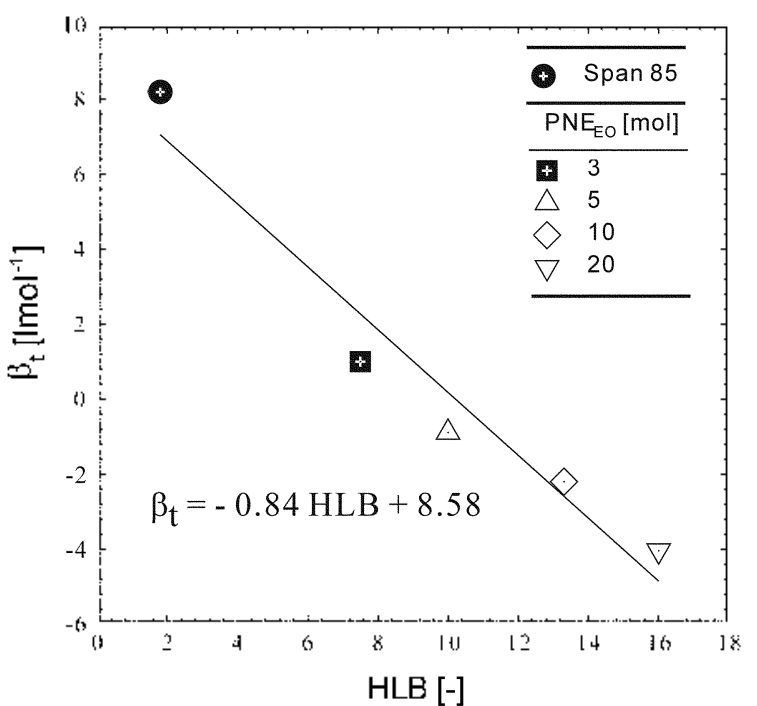

Figure 5. Correlation between the value of HLB and $\beta_{t}$ of PNL's and Span 85.

concentration added in the reverse micellar solution in Figure 3 . There is a linear correlation between $\Delta \phi_{p}$ and the concentration of each nonionic-cosurfactant. The slope, $\beta_{1}$, is a measure of the effect of nonionic-cosurfactant addition on the micellar-micellar interaction. We found that variation in these effects can be explained by the additive contribution of each constituent group of the nonionic-cosurfactant.

The $\beta_{1}$ values for various thiols and nonionic-surfactant are plotted against the values of $\log P$ and HI.B in Figure 4 and Figure 5, respectively,

It shows that the $\beta$ values are increased with increasing the values of $\log \mathrm{P}$ and decreasing the values of HLB. The $\beta$, values are increased with increasing the hydrophobicity of nonionic-surfactants. This is suggesting that the hydrophobicity of additive molecules is a key factor and respon-

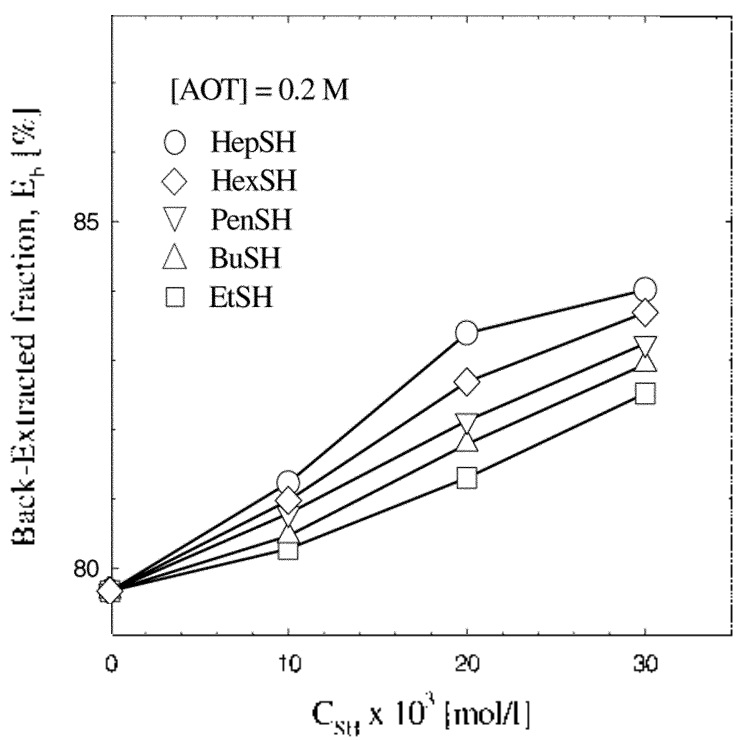

rigure 6. Leffect of thiols addition on the back-extraction fractions of c.c.lipast. 


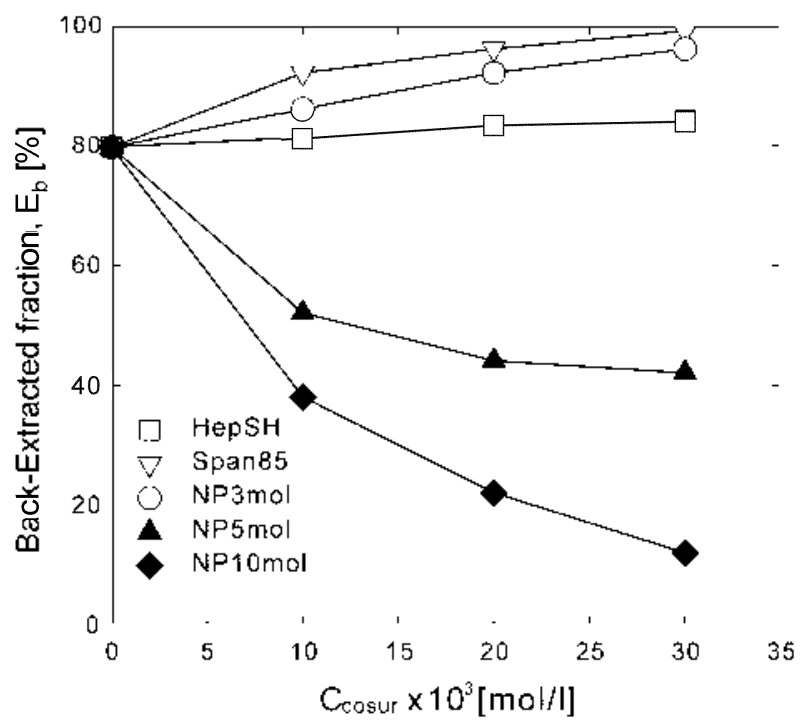

Figure 7. Eiffect of nonion surfactants addition on the hackcxtraction fractions of c.c.lipase for AOT (0.1 V)-RVMS.

sible for their effect on the suppression of the micellarmicellar interactions inducing micellar clustering.

Effect of additives addition on the back-extracted fraction and its rates of the c.c.lipase. We have examined the back-extraction of c.c.lipase using various additives at low ionic strength conditions $([\mathrm{KCl}]=0.1 \mathrm{M})$. Figure 6 and Figure 7 show the effects of various thiols and nonionic surfactants on the back-extracted fraction of c.c.lipase. respectively. The back-extracted fraction of c.c.lipase varies with the addition of a small amount of thiols and nonionic surfactants. [specially, the back-extracted fraction of c.c.lipase is increased with addition of Span 85 to almost $100 \%$ of back-extracted fraction. This result suggests that the backextraction behaviors can be improved by a small amount of Span 85 and thiols. However, PNF, with 5, 15,20 mol have an effect of reducing the back-extraction fraction. It shows that

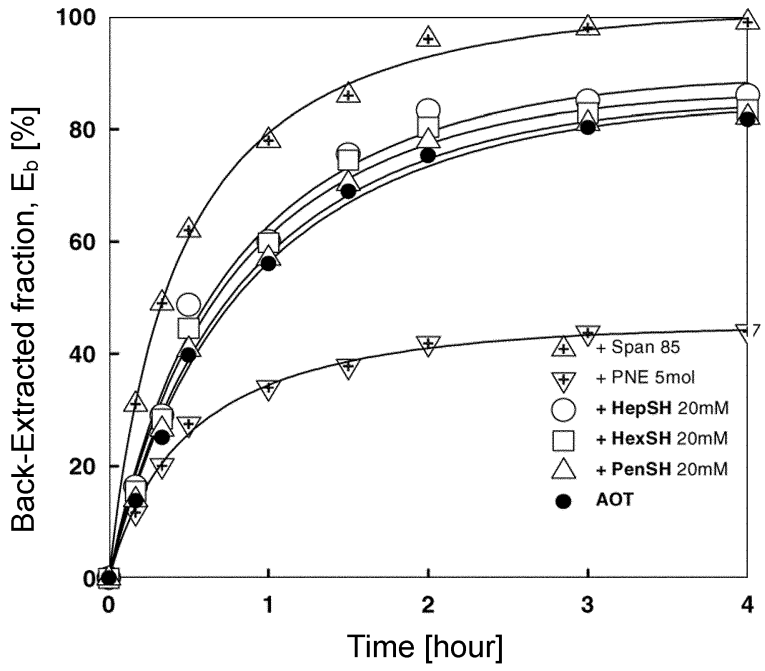

Figure 8 . Time course of the back-extracted fraction of c.c.lipase for the AOT/Additives-RVMSs (Salt concentration in stripping aqueous solution is $0.1 \mathrm{M}$ ).
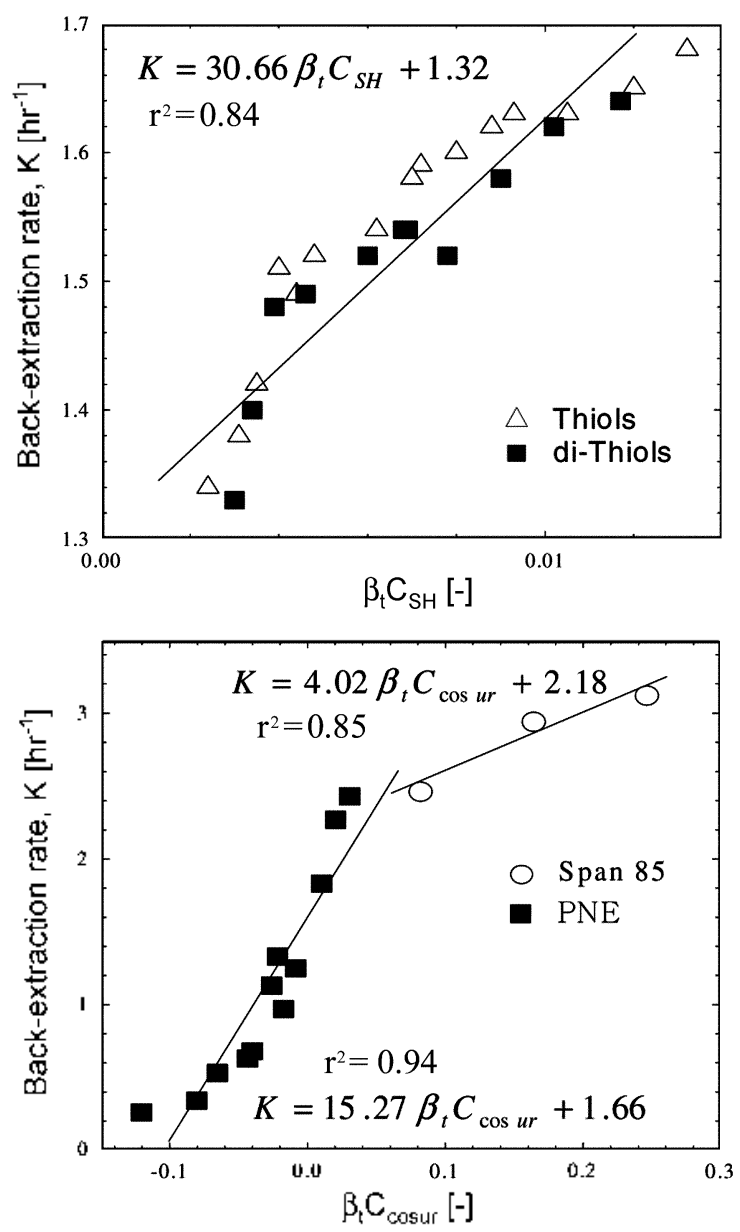

Figure 9. Correlation between the percolation processes $(\beta)$ and the rate constant of back-extraction (K) of c.c.lipase.

the increasing $\beta_{1}$ value promotes the back-extraction of c.c.lipase.

Figure 8 shows the effect of additives addition on the time course of the back-extracted fraction of c.c.lipase. There is a clear difference depending on the species of additives added to RVMS. For example, in the case of the addition of $\mathrm{P} N E_{\mathrm{EO}}$ $10 \mathrm{~mol}$, the back-extraction rate is reduced greatly. Whereas in the addition of HepSH or Span85, the back-extraction rates are accelerated remarkably. This is an interesting result indicating the possibility that the protein back-extraction process can be regulated by a small amount of additives addition to RVMS. It is considered that these variations of the back-extraction behaviors can be induced by the cosurfactant effect on the micellar-micellar interaction and c.c.lipase-Inicellar interaction.

Analysis of c.c.lipase back-extraction process in the Additives mediated AOT/isooctane RVMS. We have examined the relationship between the c.c.lipase backextraction and the percolation behaviors. The rate constant of back-extraction $(K)$ is plotted against the variation of percolation processes $\left(\beta_{t}\right)$ at the each same additives concentration in Figure 9 .

It shows that the increasing $\beta_{1}$ value promotes the backextraction rate constant. $K$. In other word, the decreasing of 
the micellar-micellar interaction accelerates the c.c.lipase back-extraction rate. explaining the role of additives to the back-extraction process of c.c.lipase in RVMS. We are considering that the reverse micelle system property is changed by addition of additives. affecting the micellarmicellar and c.c.lipase- micellar interactions.

\section{Conclusion}

The values of $\beta$ defined by the variation of percolation processes and back-extraction behaviors of c.c.lipase have good a linear correlation.

Addition of a small amounts of thiols and nonionic surfactants in an organic solution suppressed the cluster formation of reverse micelles, improved the back-extraction fraction of c.c.lipase.

The rate of back-extraction of c.c.lipase is increased with decreasing of the value of hydrophilic lipophilic balance (HLB) and increasing of the hydrophobicity per additives molecule added to RVSM in same additives concentration.

Acknowledgment. This paper was supported by the Dong-A University Research Fund in 2002

\section{References}

1. Goklen. K.: Hatton. T. A. Sci. Techmol 1987.22.831

2. Luisi, P. L.: Gionini. M: Pileni. M. P.: Robinson, B. H. Biochent. Biophys Acta $1988,974,209$.

3. Hatton. T. A. In Siffactan-Based Separation Processes; Scamehon. J. F.: Harwell. J. H.. Eds.: Marcel Dekker Press: New York.
U.S.A.. 1989: pp 55-90

4. Dekiker. M.: Vant Riet. K.: Bijsterbosch. B. H.: Wolbert. R. B. G.: Hilhorst, R. Chent. Eng. Sci. 1990. 45, 2949.

5. Dekker. M.; Riet, K. V.: Van Der Pol. J. J. Chent. Eng. 1991, t6. B69.

6. Kuboi. R.: Hashimoto. K.: Komasawa. I. Kagaku Kogaku Ronbushu 1990 a. 15.335 .

7. Kuboi. R.: Mori. Y.: Komasawa. I. Kagaku Kogaku Ronbushu 1990b. 16,763 .

8. Correa. N. M.; Durantini. E. N.: Silber. J. J. Jom of Colloid and Interface Science 2001. 240.573.

9. Shraboni. D.: Subho. M.: Amarnath. M. Jownal of Colloid and Interface Science 2000. 230.328.

10. Yasuhiro. N.: Takumi. K.: Susumu. N.: Katsuroku. T. Joumal of Mentbrane Science 2002. 195. 11.

11. Sakono, M: Goto, M.: Furusaki, S. Jommal of Bioscience and Bioengineening 2000, $89(5), 458$.

12. Goto. M.: Hashimoto. Y: Fujita. T:: Ono. T.: Furusaki. S. Biotechol. Prog. 2000. 16. 1079

13. Lee. S.-S.: Lee. B.-K.: Lee. J.-P. Bull. Korean Chem. Soc. 2001. $22(8) .897$.

14. Hong. D.-P.; Lee. S.-S.; Kuboi, R. J. Chromatogr. $B$ 2000B, $7+3$. 203.

15. Dungant. S. R.: Bausch. T.: Hatton. T. A.: Plucinski. P.: Nisch. W. J. Colloid hiterface Sci. 1991. 145.33.

16. Kuboi. R.: Hong. D. P.: Komasawa. I.: Shiomori. K.: Kawano. Y: Lee. S. S. Solv Exm Res Det: Japan 1996. 3, 223.

17. Hong. D. P: Kuboi. R.: Komasawa, I. Korem. J. Chent. Eng. 1997. $1+(5) .334$.

18. Alesandridis. P.: Holzwarth. J. F.: Hatton. T. A. J. Phus. Chem. 1995. 99. 8222

19. Larsson. K. M.: Pileni. M. P. . Eur Biophys. 1993. 21. 409.

20. Cassin. G. S.: Pileni. M. P. Chent. Ptns. Letters 1994. 221. 205.

21. Mukhopadhyay, L.: Bhattacharya, P. K.: Moulik. S. P. Colloids and Sinfaces 1990,50.295.

22. Suarez. M.-J.: Levy. H.: Lan1g. J. J. Phus. Chem. 1993. 97.9808 